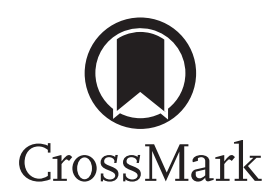

\section{Inhaled corticosteroids and increased microbial load in COPD: potential role of epithelial adhesion molecules}

\begin{abstract}
To the Editor:
We read with great interest the recent very elegant study by ConToLI et al. [1] published in the European Respiratory Journal, and the accompanying editorial by WILKInson [2], wherein authors investigated the long-term effects of inhaled corticosteroids (ICS) on microbial load in chronic obstructive pulmonary disease (COPD). It is an important area, and it is encouraging and reassuring that leading respiratory journals are recognising this. Understanding the mechanisms behind microbial infections is very important. In the discussion, the authors mentioned that the mechanisms by which these communities of different microorganisms interact with the epithelium and influence the immune system in COPD are essentially unknown. We would like to take this further, and suggest a broader discussion of new insights into the potential mechanisms contributing to increased adherence of microbes to airway epithelium and immune cell populations.
\end{abstract}

The prerequisite step is the adherence of pathogens to the respiratory mucosa. The interaction between the epithelial and bacterial surface occurs through phosphorylcholine (ChoP), a molecular mimic of platelet-activating factor (PAF) present on the bacterial surface, while PAF receptor (PAFR) is expressed on the airway epithelium [3-6]. Both airway pathogens, pneumococcus and Haemophilus influenzae can attach to airway epithelial PAFR through ChoP and remain protected from body's innate immune responses [3]. We have previously reported that PAFR expression increases in the airways of smokers and COPD patients [6,7]. Importantly in this study, we also looked at the effects of fluticasone propionate (FP) on PAFR expression in COPD patients [7]. Since ICS are known to be a major risk factor for pneumonia, we hypothesised whether ICS itself increases PAFR expression and facilitates bacterial adhesion. Interestingly, we found that high doses of FP elevated PAFR expression in COPD patients compared to placebo. The intervention, though underpowered, still provided interesting inputs on the likely cause of observed vulnerability to pneumococcus infection in COPD patients treated with FP.

Further, we also observed an increase in PAFR expression in the small airway, inflammatory cells and alveolar epithelium of COPD patients [8]. The pan-airway presence of PAFR in COPD patients is likely to provide necessary adhesion sites throughout the lung tissue, hence increasing the risk of bacterial colonisation at multiple regions [8]. Similarly, in COPD patients, we observed an epithelial increase in another such adhesion molecule, intercellular adhesion molecule 1, suggesting involvement of more than one receptor facilitating such mechanisms [5]. In an in vitro study using BEAS-2B lung epithelial cells, we further demonstrated that antagonising PAFR by using specific chemical blockers lead to significant decrease in adherence and engulfment of both nontypeable H. influenzae and Streptococcus pneumoniae in a dose-dependent manner [4]. This also suggests PAFR as potential therapeutic target in COPD to block chronic microbial colonisation and reduce acute exacerbations.

Another likely factor from our observations for the increased vulnerability of COPD patients to infections is indeed the very lack of key inflammatory cells in the airway wall of mild-moderate COPD patients [9]. The administration of ICS in such patients would reduce their ability to mount an appropriate immune response to the infection. Further, in our recently published study [10], we observed differential macrophage switching occurring in small airway wall and bronchoalveolar lavage (BAL) derived alveolar macrophages (AMs). While the nonsmokers' small airway walls, which were predominantly M2 $\left(\mathrm{CD} 163^{+}\right)$,

@ERSpublications

ICS have the potential to increase infection by upregulating epithelial microbial adhesion molecules http://ow.ly/Um7730haVgb

Cite this article as: Sohal SS. Inhaled corticosteroids and increased microbial load in COPD: potential role of epithelial adhesion molecules. Eur Respir J 2018; 51: 1702257 [https://doi.org/10.1183/13993003.022572017]. 
switched towards the $\mathrm{M} 1$ phenotype $\left(\mathrm{CD} 8^{+}\right.$inducible nitric oxide synthase (iNOS) ${ }^{+}$dual positive) in smokers and early-stage COPD, the BAL AMs switched towards a more M2 phenotype and were mainly driven by cytokines/chemokines that skewed more towards a M2/Th2 profile. Moreover, we observed that the BAL AMs from COPD patients had downregulated iNOS expression when compared to nonsmokers, again pointing towards the existence of dysfunctional BAL AMs, which we suspect could be further suppressed by ICS treatment [10]. With such intrinsic immune complexities involved, a largely nonspecific, broad-spectrum corticosteroid, we believe, will not be very efficient, and on the contrary, may produce undesired effects of an increased susceptibility to infections in COPD patients as reported by Contoli et al. [1].

Taken together, these observations suggest that microbial adhesion molecules are important epithelial attachment sites for both viruses and bacteria, which are potentially upregulated in response to ICS treatment and increase microbial load. This complex pathology is further exaggerated due to the increasingly immunocompromised situation in COPD patients. Our findings in COPD might well be applicable to other chronic lung disease such as asthma and interstitial lung diseases [3]. Translational research in this area of microbial-epithelial interactions is still in its infancy, but has a huge potential to provide novel insights into COPD. There is an essential need to rethink COPD airway pathology for identification of new therapeutic targets. This recent paper by ConTOLI et al. [1] is a timely reminder that understanding these mechanisms is of utmost importance.

Sukhwinder Singh Sohal

Dept of Laboratory Medicine, School of Health Sciences, Faculty of Health, University of Tasmania, Launceston, Australia.

Correspondence: Sukhwinder Singh Sohal, School of Health Sciences, University of Tasmania, Locked Bag - 1322, Newnham Drive, Launceston, Tasmania 7248, Australia. E-mail: sssohal@utas.edu.au

Received: Nov 012017 | Accepted: Nov 092017

Support statement: This work was supported by Clifford Craig Foundation. Funding information for this article has been deposited with the Crossref Funder Registry.

Conflict of interest: None declared.

\section{References}

1 Contoli M, Pauletti A, Rossi MR, et al. Long-term effects of inhaled corticosteroids on sputum bacterial and viral loads in COPD. Eur Respir J 2017; 50: 1700451.

2 Wilkinson TMA. Are inhaled corticosteroids increasing the "load" for some patients with COPD? Eur Respir J 2017; 50: 1701848 .

3 Sohal SS, Hansbro PM, Shukla SD, et al. Potential mechanisms of microbial pathogens in idiopathic interstitial lung disease. Chest 2017; 152: 899-900.

4 Shukla SD, Fairbairn RL, Gell DA, et al. An antagonist of the platelet-activating factor receptor inhibits adherence of both nontypeable Haemophilus influenzae and Streptococcus pneumoniae to cultured human bronchial epithelial cells exposed to cigarette smoke. Int J Chron Obstruct Pulmon Dis 2016; 11: 1647-1655.

5 Shukla SD, Mahmood MQ, Weston S, et al. The main rhinovirus respiratory tract adhesion site (ICAM-1) is upregulated in smokers and patients with chronic airflow limitation (CAL). Respir Res 2017; 18 : 6.

6 Grigg J, Walters H, Sohal SS, et al. Cigarette smoke and platelet-activating factor receptor dependent adhesion of Streptococcus pneumoniae to lower airway cells. Thorax 2012; 67: 908-913.

7 Shukla SD, Sohal SS, Mahmood MQ, et al. Airway epithelial platelet-activating factor receptor expression is markedly upregulated in chronic obstructive pulmonary disease. Int J Chron Obstruct Pulmon Dis 2014; 9: 853-861.

8 Shukla SD, Muller HK, Latham R, et al. Platelet-activating factor receptor (PAFr) is upregulated in small airways and alveoli of smokers and COPD patients. Respirology 2016; 21: 504-510.

9 Eapen MS, McAlinden K, Tan D, et al. Profiling cellular and inflammatory changes in the airway wall of mild to moderate COPD. Respirology 2017; 22: 1125-1132.

10 Eapen MS, Hansbro PM, McAlinden K, et al. Abnormal M1/M2 macrophage phenotype profiles in the small airway wall and lumen in smokers and chronic obstructive pulmonary disease (COPD). Sci Rep 2017; 7: 13392. 